\title{
Usefulness of perioperative rotational thrombelastometry during scoliosis surgery in children
}

\author{
Jakub Jonas, MD, ${ }^{1}$ Miroslav Durila, MD, PhD, ${ }^{1}$ Martin Malosek, MD, ${ }^{1}$ Dagmar Maresova, MD, ${ }^{1}$ \\ Jan Stulik, MD, PhD, ${ }^{2}$ Michal Barna, MD, ${ }^{2}$ and Tomas Vymazal, MD, PhD' \\ 'Department of Anesthesiology and Intensive Care Medicine, Second Faculty of Medicine, and ${ }^{2}$ Centre for Spinal Surgery, \\ First Faculty of Medicine, Charles University and Motol University Hospital, Prague, Czech Republic
}

\begin{abstract}
OBJECTIVE Surgical correction of scoliosis in pediatric patients is associated with significant blood loss. Rotational thrombelastometry (ROTEM) might help to decrease the use of blood transfusion products by enabling an early point of care (POC) diagnosis of coagulopathy, thus helping to provide targeted therapy. The aim of this case-control study was to find out whether POC use of ROTEM during scoliosis surgery in children helps to reduce the need for blood transfusion products.
\end{abstract}

METHODS Data were prospectively analyzed from all patients treated during 2016-2018 who received ROTEM-based therapy during scoliosis surgery. These patients were compared with a group of historical controls treated during 20142016 whose scoliosis treatment did not include ROTEM. Perioperative blood loss, consumption of blood transfusion products, and hospital LOS were compared between the groups.

RESULTS A total of 37 patients were analyzed, 22 patients in the non-ROTEM group and 15 patients in the ROTEM group. In the ROTEM group compared with the non-ROTEM group, there was significantly lower perioperative blood loss and administration of packed red blood cell units, no administration of fresh-frozen plasma, and shorter overall hospital $\operatorname{LOS}(p<0.05)$.

CONCLUSIONS ROTEM use during scoliosis surgery in children seems to help to decrease blood loss and the use of blood transfusion products and may also shorten the hospital LOS.

Clinical trial registration no.: NCT03699813 (clinicaltrials.gov).

https://thejns.org/doi/abs/10.3171/2019.11.SPINE191137

KEYWORDS rotational thrombelastometry; scoliosis surgery; blood product management; coagulation testing; pediatric surgery; deformity; diagnostic technique

$\mathrm{C}$ ORRECTION of scoliosis of the thoracic and lumbar spine in pediatric patients is an extensive surgery that is usually associated with significant blood loss. ${ }^{1,5}$ Treatment of this perioperative blood loss and coagulopathy often includes administration of blood transfusion products such as packed red blood cells (PRBC) and fresh-frozen plasma (FFP). These blood products are usually administered based on the results of laboratory tests or according to the experience of the anesthesiologist. However, the use of FFP and PRBC is associated with numerous health risks. ${ }^{11,13}$

Prothrombin time and activated partial thromboplastin time are blood plasma tests commonly used to assess the status of hemocoagulation. However, these tests are very time-consuming, taking about 45 minutes, and therefore are very rarely used for the perioperative assessment of coagulopathy. Moreover, according to the new cell-based model of hemocoagulation, ${ }^{7}$ whole blood seems to be more suitable for assessing blood clotting. Therefore, rotational thrombelastometry (ROTEM) seems to be more suitable for assessing patient hemocoagulation status during the perioperative period. ROTEM is a viscoelastic method that uses a whole-blood sample to assess the initiation and propagation phases of clot formation and clot strength and also provides information about fibrinolysis. As a point of care (POC) method, ROTEM provides the first clinically

ABBREVIATIONS FFP = fresh-frozen plasma; LOS = length of stay; $P O C=$ point of care; $P R B C=$ packed red blood cells; ROTEM = rotational thrombelastometry;

TXA = tranexamic acid.

SUBMITTED September 19, 2019. ACCEPTED November 20, 2019.

INCLUDE WHEN CITING Published online January 24, 2020; DOI: 10.3171/2019.11.SPINE191137. 
important results in around 5-10 minutes. Thus, it allows for the rapid diagnosis of coagulopathy and enables adequate targeted therapy. ${ }^{3,6,14}$

FFP is often used in clinical practice in bleeding patients. At the same time, its use is associated with significant risks, including transfusion-related acute lung injuries, transfusion-associated circulatory overload, allergic and anaphylactic reactions, transmission of infections, hemolytic reactions, febrile nonhemolytic reactions, erythrocyte alloimmunization, prolongation of hospitalization, and the development of postoperative complications with a need for a revision surgery, which may be associated with increased morbidity and mortality. ${ }^{11,13}$ In trauma patients, some studies reported that with each administered FFP transfusion unit, the risks of multiorgan failure (MOF) and acute respiratory distress syndrome (ARDS) are increased by $2.1 \%$ and $2.5 \%$, respectively ${ }_{15}^{15}$ and that the administration of FFP outside a massive transfusion protocol (10 or more FFP units administered in 12 hours) increases the incidences of ARDS, MOF, pneumonia, and sepsis 12 times, 6 times, 4 times, and 4 times, respectively. ${ }^{8}$ Therefore, there is a worldwide tendency to avoid the redundant administration of FFP and other transfusion products. It can be presumed that targeted therapy of coagulopathy based on the results of ROTEM might help to avoid a decrease in the administration of blood transfusion products.

In this study, we hypothesized that perioperative use of ROTEM might decrease the intensity of perioperative bleeding and the consumption of blood transfusion products by rapid diagnosis of coagulopathy and subsequent targeted therapy in pediatric patients undergoing scoliosis surgery.

\section{Methods}

The study was approved by the Ethics Committee for Multi-Centric Clinical Trial of the Motol University Hospital in Prague, Czech Republic, on February 4, 2015 (reference no. EK-28/15) and was registered at ClinicalTrials. gov (reference no. NCT03699813).

Pediatric patients (younger than 19 years) undergoing primary surgery for the treatment of juvenile or neuromuscular scoliosis were included in the study. Surgery was performed either in a single session by the dorsal approach or in 2 stages - the first stage was a release from the ventral approach, and the second stage was a correction from the dorsal approach. In patients who underwent 2-stage surgery, only data from the second phase of the surgery were included in the study.

Exclusion criteria were refusal of participation by the patient or his/her legal guardians and medical history of coagulation disorder.

The data from the 2 groups of pediatric patients who underwent scoliosis surgery were retrospectively analyzed. In the first group of patients, in whom ROTEM was used perioperatively to diagnose coagulopathy and guide adequate treatment (the ROTEM group), data were collected prospectively during 2016-2018. These patients were then matched with historical controls managed without ROTEM (the non-ROTEM group), who underwent surgery during 2014-2016. Since scoliosis surgery is a complex and spe- cialized procedure, which has been performed in our institution since 2002, all surgeries were performed by a stable group of experienced surgeons and anesthesiologists.

The aim of the study was to compare the following data between the 2 patient groups: 1) perioperative blood loss during the surgery and the 24-hour period afterward (blood loss in each child was calculated as a percentage of the estimated total blood volume to eliminate any possible error caused by differences in age and body weight between patients); 2) PRBC and FFP units required during the surgery and the 24-hour period afterward (calculated as $\mathrm{ml} / \mathrm{kg}$ of body weight); and 3) total length of stay (LOS) in the pediatric intensive care unit (PICU) and in the hospital before discharge.

In the ROTEM group, ROTEM (ROTEM delta machine, Tem International $\mathrm{GmbH}$ ) was used for the evaluation of hemostasis in the operating room as well as in the PICU. The following tests were used: EXTEM (contains recombinant tissue factor, which activates the extrinsic coagulation pathway), INTEM (contains partial thromboplastin, which activates the intrinsic coagulation pathway), FIBTEM (contains cytochalasin D, which inhibits platelet function for the evaluation of the function of fibrinogen), and APTEM (contains aprotinin, which inhibits fibrinolysis in vitro, to confirm suspected fibrinolysis on the EXTEM test).

The following parameters were evaluated in the EXTEM and INTEM assays: 1) clotting time (CT) from the start of measurement until the first fibrin formation detected at an amplitude of $2 \mathrm{~mm}$, which represents the initiation phase of clotting; 2) clotting formation time (CFT) from CT until clot firmness detected at an amplitude of 20 $\mathrm{mm}$, the value that represents fibrin polymerization and clot stabilization with platelets and fibrinogen; 3) alpha angle, the angle between the time axis and the ROTEM curve, which together with CFT represent the propagation phase of clot formation and its kinetics; 4) amplitude (A), the clot firmness in each minute from CT time; A5, A10, and A20 are the amplitudes at 5, 10, and 20 minutes, respectively; 5) maximum clot firmness (MCF); and 6) lysis index (LI), for which LI30 and LI60 represent reductions in the clot firmness in relation to MCF at 30 and $60 \mathrm{~min}$ utes from CT. A5, A10, and MCF parameters were evaluated in the FIBTEM assay.

In the ROTEM group all patients were treated on the basis of the results of ROTEM by using the protocol shown in Fig. 1. ${ }^{4}$

In the non-ROTEM group bleeding and suspected coagulopathy management was based on anesthesiologist experience and laboratory coagulation tests, platelet counts, and hemoglobin levels. Transfusion of PRBC and FFP was in a ratio of 1:1, and platelets were administered when platelet counts were below $50-100 \times 10^{9} / \mathrm{L}$.

Administration of tranexamic acid (TXA) (15-20 mg/ $\mathrm{kg}$ ), the standard of care in our department since 2014, was used in all patients in both groups at the beginning of the surgery. Another dose of TXA was administered on the basis of the ROTEM protocol.

\section{Statistics}

Statistica 13.0 software (TIBCO Software Inc.) was 


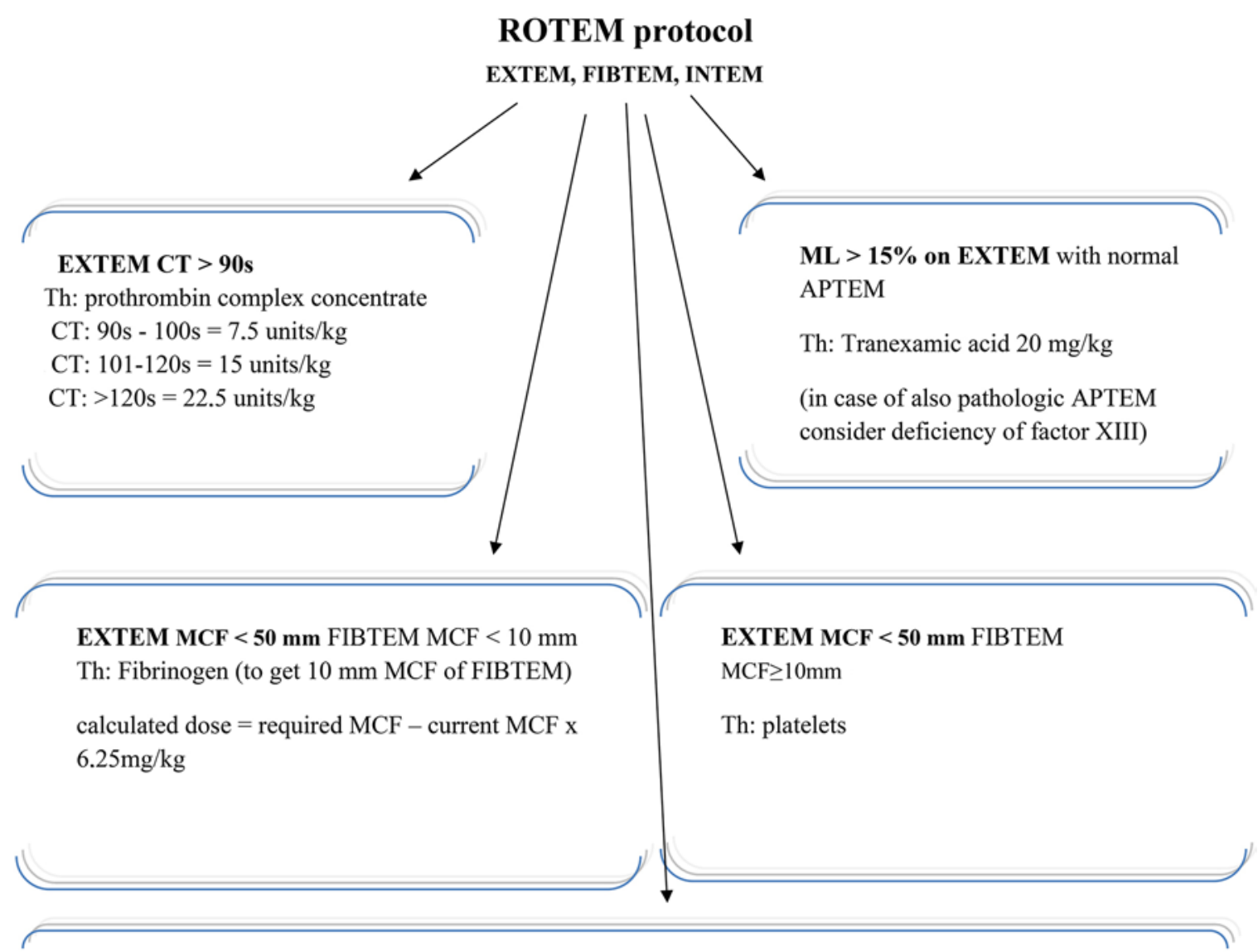

EXTEM MCF $<30-35 \mathrm{~mm}$ :

$\mathrm{Th}$ : administer all mentioned products (tranexamic acid, fibrinogen, platelets, prothrombin complex)

FIG. 1. ROTEM protocol for perioperative bleeding management. In case of normal EXTEM and FIBTEM findings in a bleeding patient, INTEM should also be performed. ML = maximum lysis; $s$ = seconds; Th = therapy. Figure is available in color online only.

used for statistical analyses. A descriptive statistic was used for each variable: count, median or mean value, standard deviation, minimum and maximum values, and lower and upper quartiles. The statistical assessment of the significance of the differences between the compared groups was performed using an unpaired Mann-Whitney U-test. A $p$ value of $<0.05$ was considered significant.

\section{Results}

Thirty-seven patients were analyzed in this study. Fifteen patients were analyzed in the ROTEM group and 22 patients were analyzed in the non-ROTEM group. The patient and surgery characteristics are presented in Table 1. Neither group statistically differed in age, sex, body weight, duration of the surgery, or number of instrumented spinal segments, and osteotomies were not done in either group. In the ROTEM group, no FFP was administered during the whole perioperative period and LOS was also shorter in the PICU as well as in the hospital ( $\mathrm{p}<0.05$, Table 2).

Blood loss was significantly lower during surgery and the 24-hour period after surgery in the ROTEM group (Fig. 2). The consumption of PRBC was also lower in the ROTEM group, and the difference was statistically significant during the 24-hour period after surgery and in overall PRBC consumption (Fig. 3). No plasma derivatives (fibrinogen, prothrombin complex concentrate) and no platelets were used in either group. There were no patient deaths or severe complications requiring surgical revision during the study.

\section{Discussion}

The results of our study suggest that the use of ROTEM has a place in the perioperative period, especially in extensive surgical procedures such as scoliosis surgery in pediatric patients. It seems that by rapid diagnosis of coagulopathy, which enables targeted therapy, the use of ROTEM-guided blood product administration may lead to reductions in perioperative blood loss and the need for blood transfusion products. Our results are similar to those reported by Guan et al., who performed a similar study in adult patients undergoing surgery for thoracolumbar 
TABLE 1. Characteristics of patients and surgery

\begin{tabular}{lccc}
\hline & & & $p$ \\
& Non-ROTEM & ROTEM & Value \\
\hline No. of patients & 22 & 15 & \\
\hline Sex & & & $>0.05$ \\
\hline Male & $5(23 \%)$ & $4(27 \%)$ & \\
\hline Female & $17(77 \%)$ & $11(73 \%)$ & \\
\hline Age, yrs & $14.4 \pm 2.3$ & $16.3 \pm 3.5$ & $>0.05$ \\
\hline Body weight, kg & $53.6 \pm 11.7$ & $64.5 \pm 17.4$ & $>0.05$ \\
\hline Lenke subtype, $\%$ & & & \\
\hline 1 & $50 \%$ & $61 \%$ & \\
\hline 2 & $17 \%$ & $8 \%$ & \\
\hline 3 & $0 \%$ & $15 \%$ & \\
\hline 5 & $28 \%$ & $8 \%$ & \\
\hline 6 & $5 \%$ & $8 \%$ & \\
\hline Preoperative Cobb angle, ${ }^{\circ}$ & $53.8 \pm 12.4$ & $53.8 \pm 10.6$ & $>0.05$ \\
\hline Surgery duration, mins & $212.4 \pm 82.9$ & $226.6 \pm 55.4$ & $>0.05$ \\
\hline Instrumented spinal segments, $\mathrm{n}$ & $10.3 \pm 2.4$ & $10.3 \pm 2.2$ & $>0.05$ \\
\hline
\end{tabular}

Values are expressed as number of patients (\%) unless otherwise indicated. Mean values are presented $\pm S D$.

deformity and found that the data provided by ROTEM analysis helped to decreased total blood product consumption during overall hospitalization. ${ }^{5}$ In addition, studies of the use of ROTEM-guided blood product administration reported by Buell et al. and Naik et al. show that ROTEMbased management of perioperative bleeding and coagulopathy reduces perioperative blood loss and use of FFP and PRBC. ${ }^{2,9}$ On the other hand, these authors reported higher consumption of cryoprecipitate. Thus, ROTEM seems to help in early identification of coagulopathy, especially low levels of fibrinogen, which is associated with increased blood loss. There are limited available data regarding the use of ROTEM in pediatric spine surgery, and this study might serve as a pilot study for other researchers and clinicians.

The mechanisms of coagulopathy in scoliosis surgery have not been satisfactorily clarified, as suggested by Bosh et al. Dilution of coagulation factors and platelets by fluid infusions and the development of fibrinolytic processes leading to consumption of coagulation factors may play roles in the development of coagulopathy. At the same time, Bosh et al. have shown that due to a stress response to the surgery, patients tend to go into a hypercoagulable state that compensates for the consumption of coagulation factors; thus, in the end there is no failure of hemocoagulation. ${ }^{1}$ This response could explain the normal ROTEM results in the ROTEM group, in which therefore no administration of coagulation factors was needed, including FFP.

In addition to the abovementioned side effects of blood products, the extensive use of FFP is also associated with undesirable hemodilution, leading to thrombocytopenia and decreased hematocrit that result in increased hemo-
TABLE 2. Differences between the non-ROTEM and ROTEM groups

\begin{tabular}{lccc}
\hline & Non-ROTEM & ROTEM & $p$ Value \\
\hline FFP consumption, $\mathrm{ml} / \mathrm{kg}$ & & & \\
\hline Surgery & $9.6 \pm 6.8$ & 0 & $<0.001$ \\
\hline $24 \mathrm{hr}$ & $11.1 \pm 7.0$ & 0 & $<0.001$ \\
\hline LOS, days & & & \\
\hline PICU & $1.6 \pm 0.7$ & $1.1 \pm 0.4$ & $<0.05$ \\
\hline Hospital & $11.7 \pm 3.7$ & $8.6 \pm 2.5$ & $<0.05$ \\
\hline
\end{tabular}

Values are presented as mean \pm SD unless otherwise indicated. Boldface type indicates statistical significance.

coagulation, bleeding, and need for PRBC administration. ${ }^{3}$ These adverse effects of FFP administration may be associated with worse patient outcomes, with increased morbidity and hospital LOS, as reported by Purvis et al. ${ }^{12}$ These factors might explain the results in the nonROTEM group in the our study, including increased blood loss and consumption of PRBC and FFP and longer PICU and hospital LOS.

Similar results, such as reduced blood loss and PRBC consumption and the shortening of LOS in the ICU, were also observed by Nakayama et al. in their study of the use of the ROTEM method in pediatric cardiac surgery. ${ }^{10}$ However, there are limited data for ROTEM use in other pediatric surgical fields, and we hope that this pilot study will help other authors to perform clinical studies on the same topic.

\section{Study Limitations}

Limitations of the study include its retrospective nature and small number of patients. However, data describing the use of ROTEM in the perioperative period in spine surgery are limited, especially in children. That is why this study may serve as a pilot study and may help other researchers and clinicians to perform similar studies to support these data. Despite the relatively small number of patients, it was possible to get statistically and clinically significant results that are important for clinical practice. Moreover, similar results have already been observed in adult patients with comparable study group sizes (15 patients in the ROTEM group and 15 patients in the nonROTEM group). ${ }^{5}$

Other limitations may be that TXA was administered to all patients in both the ROTEM and non-ROTEM groups, as it is standard for our department. On the other hand, regarding this point we can assess both groups as being homogenous.

\section{Conclusions}

Our data support using ROTEM as a POC method for perioperative use in pediatric patients undergoing scoliosis surgery. The fast results provided by ROTEM help anesthesiologists to administer targeted therapy and prevent the administering of redundant coagulation factor products, including FFP. Rapid diagnosis and subsequent treatment of coagulopathy may also help to decrease peri- 

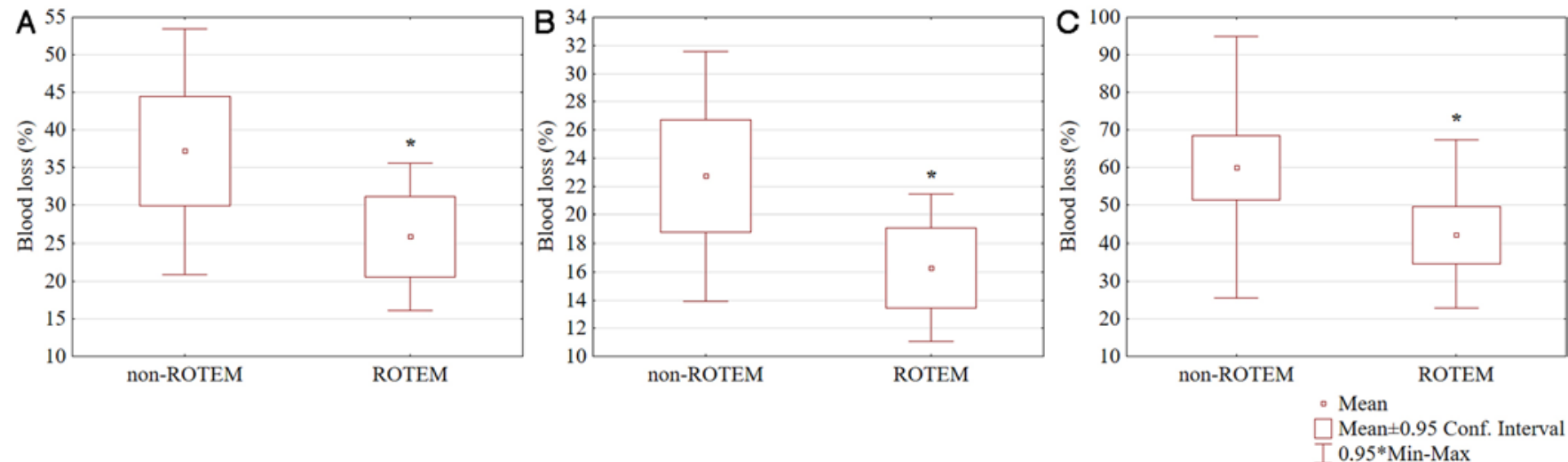

FIG. 2. Blood loss (\% of estimated total blood volume) during surgery (A), the 24 -hour period after surgery (B), and overall (C). ${ }^{*} p<0.05$. Figure is available in color online only.
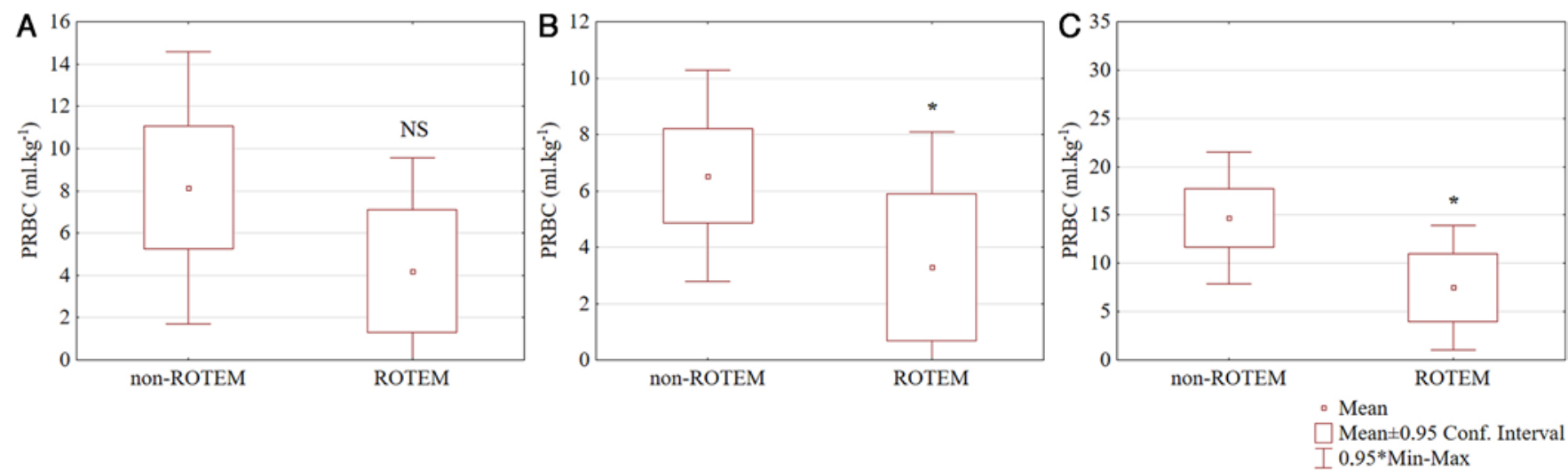

FIG. 3. PRBC consumption ( $\mathrm{ml} / \mathrm{kg}$ body weight) during surgery (A), the 24 -hour period after surgery (B), and overall (C). ${ }^{*} p<0.05$. NS = nonsignificant. Figure is available in color online only.

operative blood loss and the consumption of PRBC and FFP, which seem to be associated with shorter PICU and hospital LOS.

\section{Acknowledgments}

We thank Jackson Jones for language correction of the text. This study was supported by the Ministry of Health, Czech Republic-Conceptual Development of Research Organization, Motol University Hospital, Prague, Czech Republic (no. 00064203).

\section{References}

1. Bosch P, Kenkre TS, Londino JA, Cassara A, Yang C, Waters $\mathrm{JH}$ : Coagulation profile of patients with adolescent idiopathic scoliosis undergoing posterior spinal fusion. J Bone Joint Surg Am 98:e88, 2016

2. Buell TJ, Taylor DG, Chen CJ, Dunn LK, Mullin JP, Mazur $\mathrm{MD}$, et al: Rotational thromboelastometry-guided transfusion during lumbar pedicle subtraction osteotomy for adult spinal deformity: preliminary findings from a matched cohort study. Neurosurg Focus 46(4):E17, 2019

3. Deng Q, Hao F, Wang Y, Guo C: Rotation thromboelastometry (ROTEM) enables improved outcomes in the pediatric trauma population. J Int Med Res 46:5195-5204, 2018

4. Durila M, Malošek M: Rotational thromboelastometry along with thromboelastography plays a critical role in the management of traumatic bleeding. Am J Emerg Med 32:288. e1-288.e3, 2014

5. Guan J, Cole CD, Schmidt MH, Dailey AT: Utility of intraoperative rotational thromboelastometry in thoracolumbar deformity surgery. J Neurosurg Spine 27:528-533, 2017

6. Haas T, Spielmann N, Mauch J, Madjdpour C, Speer O, Schmugge M, et al: Comparison of thromboelastometry $\left(\right.$ ROTEM $\left.^{\circledR}\right)$ with standard plasmatic coagulation testing in paediatric surgery. Br J Anaesth 108:36-41, 2012

7. Hoffman M, Monroe DM III: A cell-based model of hemostasis. Thromb Haemost 85:958-965, 2001

8. Inaba K, Branco BC, Rhee P, Blackbourne LH, Holcomb JB, Teixeira PG, et al: Impact of plasma transfusion in trauma patients who do not require massive transfusion. J Am Coll Surg 210:957-965, 2010

9. Naik BI, Pajewski TN, Bogdonoff DI, Zuo Z, Clark P, Terkawi AS, et al: Rotational thromboelastometry-guided blood product management in major spine surgery. J Neurosurg Spine 23:239-249, 2015

10. Nakayama Y, Nakajima Y, Tanaka KA, Sessler DI, Maeda $\mathrm{S}$, Iida J, et al: Thromboelastometry-guided intraoperative haemostatic management reduces bleeding and red cell transfusion after paediatric cardiac surgery. Br J Anaesth 114:91-102, 2015

11. Pandey S, Vyas GN: Adverse effects of plasma transfusion. Transfusion 52 (Suppl 1):65S-79S, 2012

12. Purvis TE, Goodwin CR, De la Garza-Ramos R, Ahmed 
AK, Lafage V, Neuman BJ, et al: Effect of liberal blood transfusion on clinical outcomes and cost in spine surgery patients. Spine J 17:1255-1263, 2017

13. Seicean A, Alan N, Seicean S, Neuhauser D, Weil RJ: The effect of blood transfusion on short-term, perioperative outcomes in elective spine surgery. J Clin Neurosci 21:15791585,2014

14. Sharp G, Young CJ: Point-of-care viscoelastic assay devices (rotational thromboelastometry and thromboelastography): a primer for surgeons. ANZ J Surg 89:291-295, 2019

15. Watson GA, Sperry JL, Rosengart MR, Minei JP, Harbrecht BG, Moore EE, et al: Fresh frozen plasma is independently associated with a higher risk of multiple organ failure and acute respiratory distress syndrome. J Trauma 67:221-230, 2009

\section{Disclosures}

The authors report no conflict of interest concerning the materials or methods used in this study or the findings specified in this paper.

\section{Author Contributions}

Conception and design: Durila, Jonas, Vymazal. Acquisition of data: Durila, Jonas, Malosek, Maresova, Vymazal. Analysis and interpretation of data: Jonas. Drafting the article: Durila, Jonas, Vymazal. Critically revising the article: Stulik, Barna. Statistical analysis: Jonas. Administrative/technical/material support: Malosek, Maresova, Stulik, Barna.

\section{Correspondence}

Miroslav Durila: Charles University and Motol University Hospital, Prague, Czech Republic.miroslav.durila@fnmotol.cz. 